\title{
Alterations to the bronchial and bronchiolar surfaces of adult mice after exposure to high concentrations of oxygen
}

\author{
HIDEFUMI OBARA, MASAKO SEKIMOTO, AND SEIZO IWAI \\ From the Department of Anaesthesia, Kobe University School of Medicine, Ikutaku, Kobe, Japan
}

ABSTRACT We have studied the effects of inhalation of 95 to $100 \%$ oxygen on the surface morphology of the bronchi and bronchioles of adult mice, using scanning electron microscopy.

Denudation of cilia and truncation of the remaining cilia were commonly observed during high oxygen exposure. Bleb formation, a "moth-eaten" appearance of the non-ciliated cell surface and desquamation of unidentified cells were present in the bronchus after 96 hours of oxygen exposure. Deformation of non-ciliated cells, denudation of cilia, and flattening of the luminal surface were also seen in the bronchiole.

For studying the recovery process from acute oxygen damage, we returned mice to room air after four days of high oxygen inhalation and killed them four, seven and 15 days after. There were more variable and complex changes of surface morphology in individual mice during the recovery phase. We observed flattening of the epithelial surface in the bronchiole and bronchus, and noticed that deformation of non-ciliated cells were seen even after 15 days.

High oxygen inhalation causes severe morphological changes in the bronchial and bronchiolar mucosa, and those morphological lesions remain for at least two weeks after cessation of oxygen inhalation.

There are many reports on the reactions of the lung after high oxygen exposure in various experimental animals (Kistler et al, 1967; Kaplan et al, 1969; Adamson et al, 1970; Norman et al, 1971), and some attention has recently been paid to changes in the bronchial and bronchiolar mucosa after high oxygen exposure.

Several reports (Northway et al, 1967, 1969; Harrison et al, 1970; Ludwin et al, 1974) have suggested that high oxygen exposure may cause severe bronchial and bronchiolar damage in experimental animals and also in hyaline membrane disease of the neonate, which may lead to bronchopulmonary dysplasia.

In the present investigation we studied the bronchial and bronchiolar surface morphology of adult mice using scanning electron microscopy after four days of high oxygen exposure.

\section{Methods}

Eighty DDY albino male mice, weighing 20-30 g, were allocated at random into three groups $(1,2$, and 3). In group 1 ten mice breathing room air were kept in the atmospheric chamber for seven days and killed for histological preparation.

In group 230 mice were exposed to humidified $95-100 \%$ oxygen in the atmospheric chamber for four days. Only those mice surviving the scheduled exposure for 48 hours and 96 hours were used for histological preparation.

In group 340 mice were exposed to humidified $95-100 \%$ oxygen in the atmospheric chamber for four days. Oxygen concentration was then gradually lowered over half a day before the survivors were exposed to room air. The mortality rate increased considerably after the return to room air, all deaths of the exposed mice taking place within three days of return. The mortality rate was $35 \%$ in group 3 . On days four, seven, and 15 after returning to room air, mice were killed for histological preparation.

All mice were kept continuously exposed to either oxygen or room air in an acrylic chamber. The carbon dioxide produced by the animals was washed out by the flow of gas or was eliminated 
by an absorber (soda lime) attached to the bottom of the chamber. The concentration of oxygen and $\mathrm{CO}_{2}$ were monitored frequently with a Beckman $\mathrm{O}_{2}$ analyser and an infrared $\mathrm{CO}_{2}$ analyser. The $\mathrm{CO}_{2}$ concentration was less than $0.5 \%$. Room temperature was maintained between $22^{\circ} \mathrm{C}$ and $24^{\circ} \mathrm{C}$. The relative humidity was over $80 \%$. The mice were fed and were given water ad libitum.

The animals were deeply anaesthetised by an intraperitoneal injection of pentobarbital sodium. After opening the chest, the collapsed lungs were fixed by distension at a pressure of $10 \mathrm{cmH}_{2} \mathrm{O}$ $(0.98 \mathrm{kPa})$ with $2.5 \%$ buffered glutaraldehyde through a tracheal cannula. After ligation of the trachea, the heart and lungs were removed en bloc from the chest and were submerged in the same fixative for half an hour. Minute pieces of lung were fixed overnight in buffered $2.5 \%$ glutaraldehyde and postfixed for an hour in buffered $1 \%$ $\mathrm{OsO}_{4}$. Blocks of lungs were dehydrated with graded ethanol and cleared of carbon dioxide using the critical point method (Hitachi-HCP-I). The dried samples were mounted on aluminium stabs and coated with carbon and gold, using an Akashi vacuum rotary evaporator. Samples were examined under a Hitachi-SSM-II scanning electron microscope at $20 \mathrm{kv}$. Blocks of lungs were also stained with haematoxylin and eosin for light microscopy.

\section{Results}

Control Lung-Two distinctive cell types, ciliated and non-ciliated, were observed on the mucosal surface of bronchi. In the main bronchi ciliated cells were covered with many short microvilli which intermingled with a long complex of cilia leaning slightly in a certain direction. Non-ciliated cells were not protruded as compared to the neighbouring ciliated cells and were covered with tiny microvilli (figs 1 and 2). Non-ciliated cells in the segmental bronchi, which had ragged surfaces with sulci and pits, were variable in height and interspersed among the ciliated cells. All the nonciliated cells were protruded further into the lumen than the plasmalemma of neighbouring ciliated cells (fig 3). The bronchiolar surface, at lower magnification, appeared pebbly (fig 4). The epithelium of the bronchiole was composed of ciliated cells and non-ciliated cells (Clara cells). Non-ciliated cells were protruded into the lumen and became more numerous as the diameter of the airway decreased. Non-ciliated cells predominated over most of the surface, and ciliated cells were interspersed among non-ciliated cells. The surfaces of the non-ciliated cells were relatively smooth, with small and sparse microvilli. Cilia in the

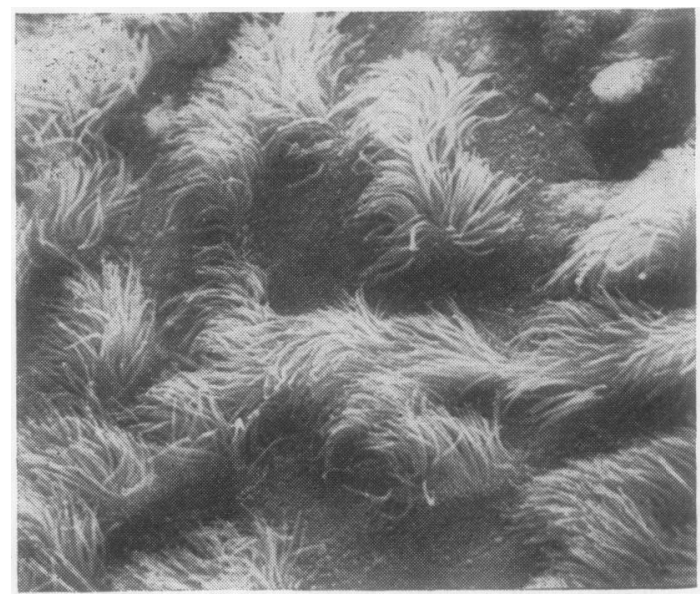

Fig 1 Main bronchus of control mouse. Ciliated cells with array of long cilia and non-ciliated cells which are not protruded compared to neighbouring ciliated cells and which are covered with tiny microvilli on surface $(\times 2000)$.

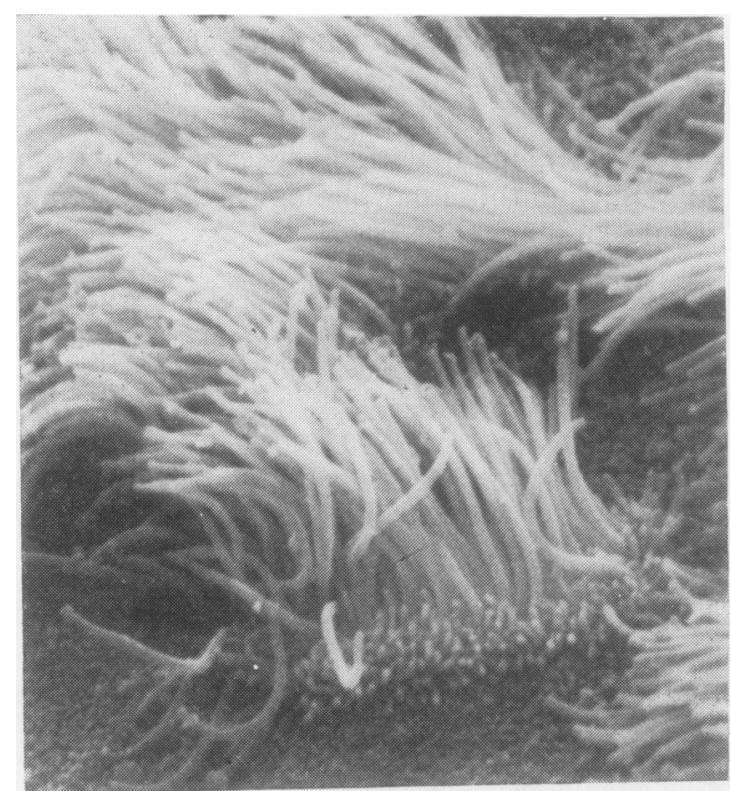

Fig 2 Main bronchus of control mouse. Ciliated cells covered with a long complex of cilia leaning slightly in a certain direction. Many tiny microvilli are interspersed at the base of the long cilia $(\times 5000)$

bronchiole were relatively sparse in number and $\stackrel{\circ}{\circ}$ shorter in height than those in the bronchi. The terminal bronchiole merged with the alveolar 


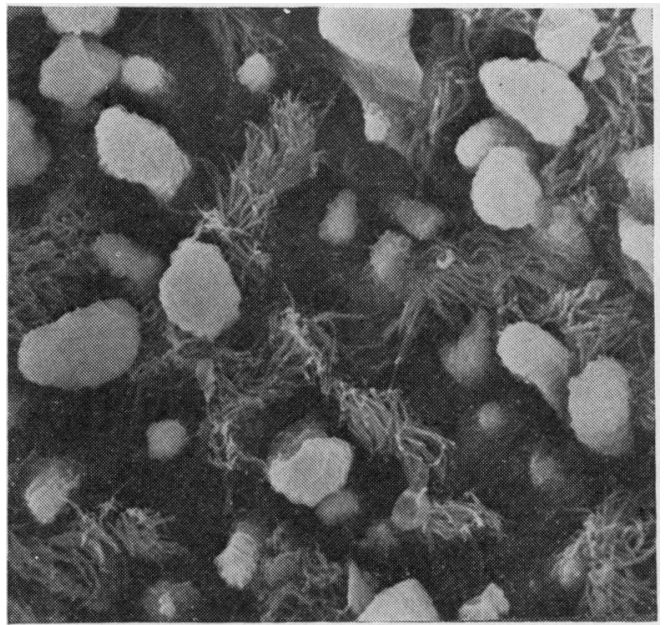

Fig 3 Segmental bronchus of control mouse. Non-ciliated cells varying in height and size are interspersed among ciliated cells. Non-ciliated cells usually protrude further into lumen than cilia $(\times 2000)$.

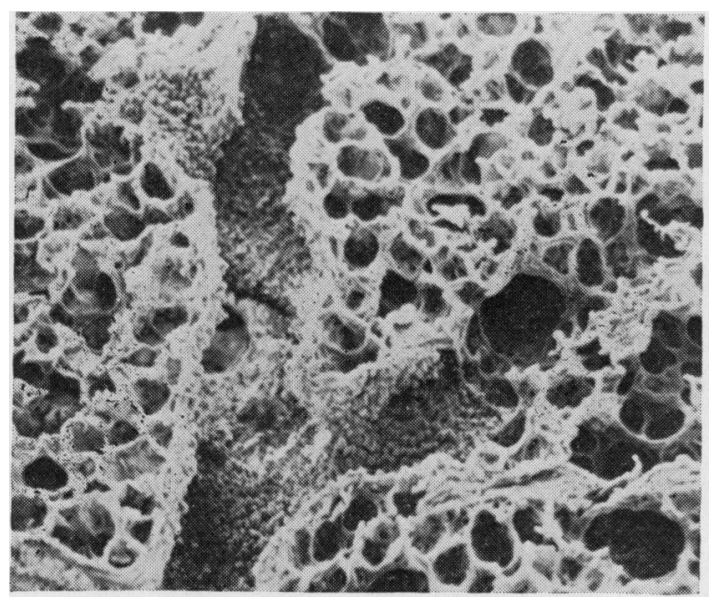

Fig 4 Terminal bronchiole, alveolar ducts, and alveoli can be seen. Surface of terminal bronchiole appears pebbly $(\times 500)$.

ducts. Little respiratory bronchiole was evident. Small alveolar pores were seen (fig 5).

After 48 hours of oxygen exposure-The surface structure of bronchi and bronchioles became more even in appearance. Denudation of cilia was the most specific change in this stage, but many short microvilli, which intermingled with long cilia, remained. The remaining cilia were truncated (fig 6).

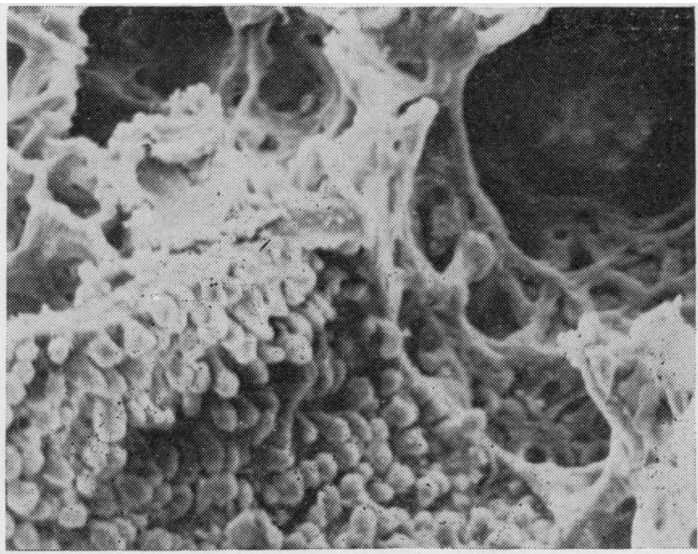

Fig 5 Terminal bronchiole merges abruptly into alveolar duct and small alveolar pores are visible $(\times 1000)$.

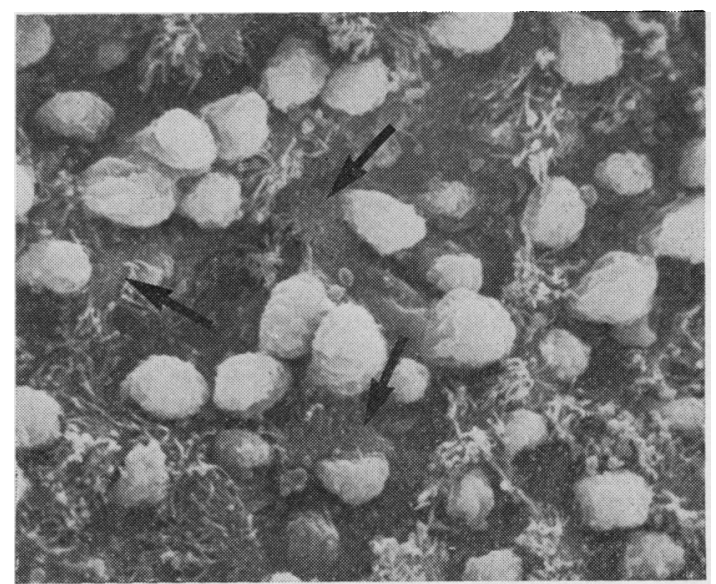

Fig 6 Segmental bronchus after 48 hours of oxygen exposure. Denudation of cilia (arrows). Remaining cilia are truncated $(\times 2000)$.

After 96 hours of oxygen exposure-These changes became more definite after 96 hours of oxygen exposure. The segmental bronchi became more even in appearance, with denudation and shortening of cilia, and also much unidentified cell debris was seen on the surface (fig 7). The nonciliated cells appeared short and deformed, and oedema of the cytoplasm of the neighbouring ciliated cells was seen by light microscopy. Many blebs with pedicles, which were usually seen in the ciliated cells with truncated and short cilia, were present in the other segmental bronchi and these blebs were seen even in the peripheral bronchi. The surface of the apical portions of the nonciliated cells, in which the cytoplasm showed 


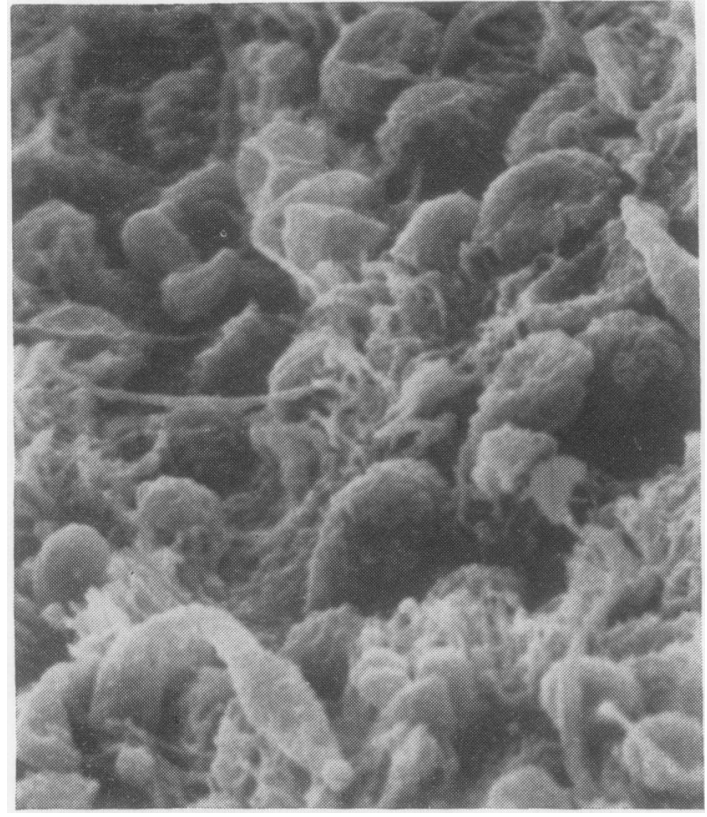

Fig 7 Segmental bronchus after 95 hours of oxygen exposure. Surface becomes more even in appearance. Many unidentified cells are scattered on surface $(\times 3000)$.

oedema and swelling by light microscopy, became flat and "moth-eaten" in appearance (fig 8). These protrusions with a moth-eaten appearance were also seen on the bronchiolar surface, where only

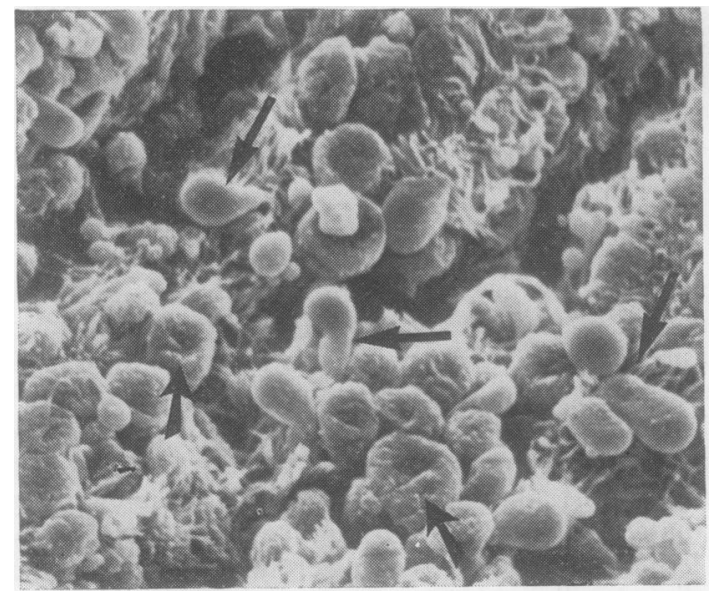

Fig 8 Another segmental bronchus after 96 hours of oxygen exposure. Many blebs with pedicles are seen (arrows). A pices of non-ciliated cells have become flat and show moth-eaten appearance (lower arrows $)(\times 2000)$. a few cilia remained. Light microscopy showed the deformation of non-ciliated cells protruding into the bronchial and bronchiolar lumen to be a degenerative process.

On the fourth and seventh days after return to room air-The remnants of ruptured bleb membranes, which filled the intercellular spaces, were observed in the segmental bronchi. The surface of the apical portions of non-ciliated cells had become flattened and more ragged than the controls. The intercellular spaces were very narrow and most cilia had been lost (fig 9). The bronchiolar surfaces were also flattened and intercellular spaces were obscure. The white-capped surfaces of the non-ciliated cells were striking in appearance, probably representing the regenerating process (fig 10). By light microscopy, the mucosal layer with distorted nuclei showed the characteristic moth-eaten appearance, and there were also migrations of pyknotic nuclei towardsthe bronchial and bronchiolar lumen. These degenerative, necrotic, and, in parts, regenerative changes in the bronchial and bronchiolar mucosal cells were intermingled in the same lung preparation at this stage.

On the 15th day after exposure to room airThe non-ciliated cells became more numerous than in the controls. Rounding of the apical portions of the non-ciliated cells with a relatively smooth surface and a uniform height was seen

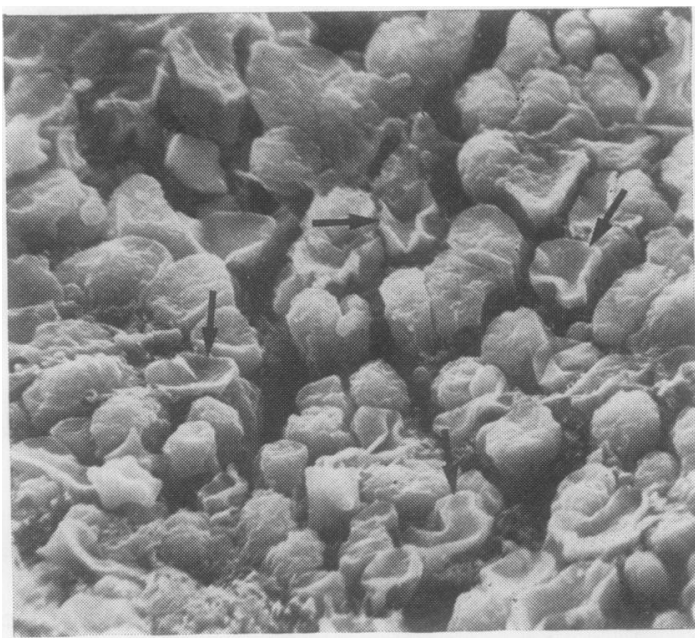

Fig 9 Four days after return to room air. Remnants of ruptured bleb membranes fill intercellular spaces in a segmental bronchus (arrows). Surfaces of apical portions of non-ciliated cells have become flat and more ragged. Intercellular spaces are very narrow $(\times 2000)$. 


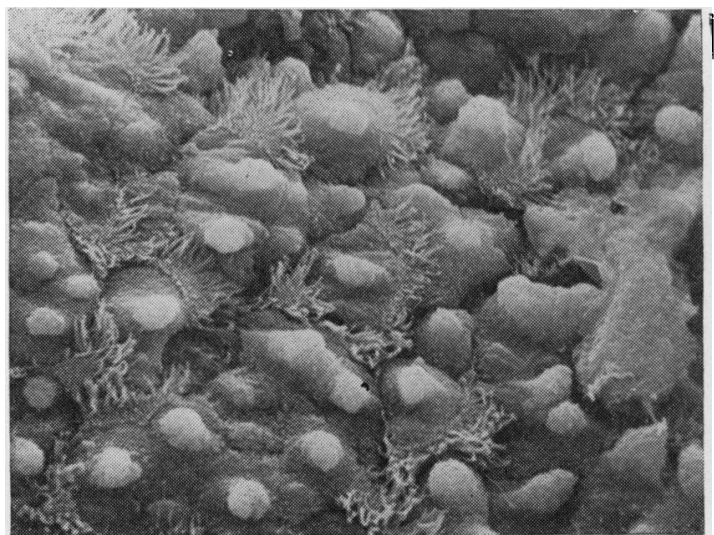

Fig 10 Seven days after return to room air. Bronchiolar surfaces are flattened and intercellular spaces are obscure in most areas. White-capped surfaces of non-ciliated cells are striking ( $\times 2000)$.

in the segmental bronchi. Only a few cilia, though without a filamentaneous appearance and shorter than in the controls, were found occasionally among the non-ciliated cells (fig 11). By light microscopy, there was scattered hyperplasia of epithelial cells in some segmental bronchi. Cuboidal cells with flattened and ragged surfaces, which differed from the control non-ciliated cells, were seen on the bronchiolar surfaces. Only a few cilia were seen (fig 12). These cells were seen to be regenerating cells with hyperchromatic, large nuclei on light microscopy.

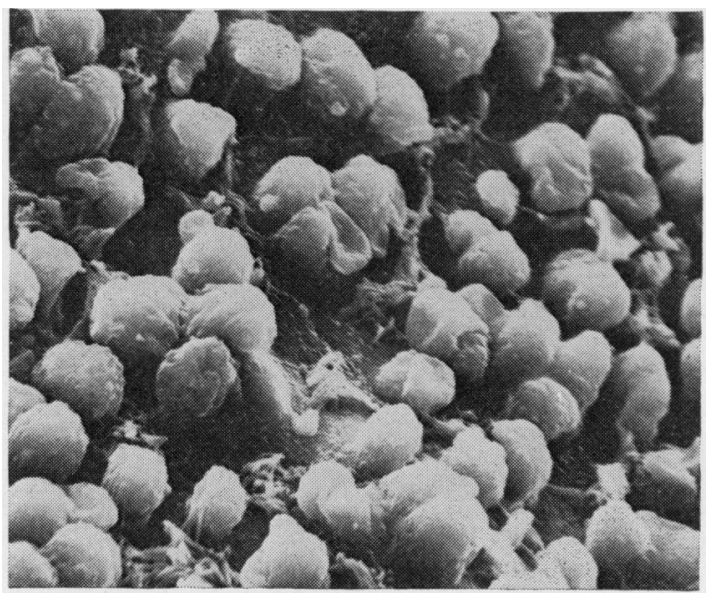

Fig 11 Fifteen days after exposure to room air. In segmental bronchus many non-ciliated cells show rounding of apical portions. Only a few cilia are scattered among non-ciliated cells $(\times 2000)$.

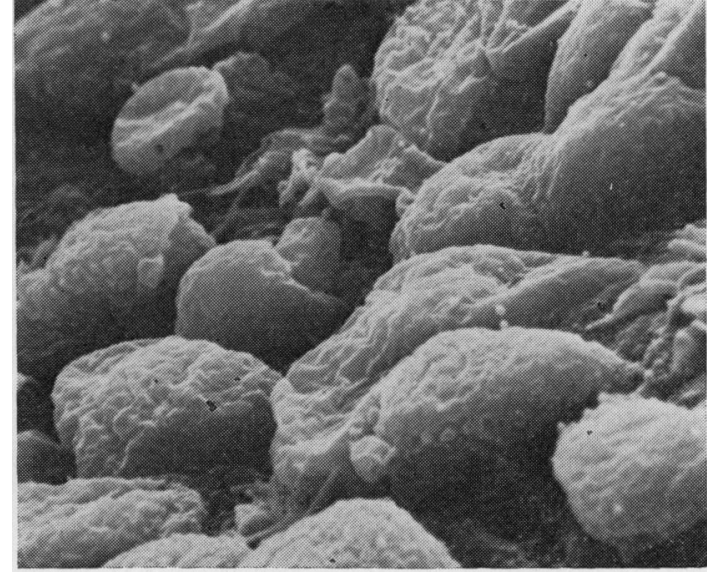

Fig 12 Fifteen days after exposure to room air. Cuboidal cells with flattened and ragged surfaces are seen in bronchiolar lumen $(\times 5000)$.

\section{Discussion}

The acute and reparative changes after exposure to high concentrations of oxygen have been investigated in many different experimental animals (Kistler et al, 1967; Kaplan et al, 1969; Adamson et al, 1970; Norman et al, 1971). Toxic effects of high concentrations of oxygen on the airway epithelium, especially the bronchi and bronchioles, have recently attracted much attention and several reports suggest that exposure to high concentrations of oxygen may cause severe bronchial and bronchiolar damage in experimental animals (Harrison et al, 1970; Ludwin et al, 1974). Postmortem studies of bronchial and bronchiolar epithelium have shown lesions that are closely related to the toxic effects of oxygen inhalation (Northway et al, 1967, 1969; Anderson and Strickland, 1971; Banerjee et al, 1972). We have carried out this study to examine the pathogenesis of pulmonary oxygen toxicity, commonly known as bronchopulmonary dysplasia, by means of surface morphology.

Pathological findings after exposure of the lung to high concentrations of oxygen are classified into exudative and proliferative phases (Nash et al, 1967). Such phases are also found on the epithelium of the oxygen-treated newborn infant (Tsai et al, 1972).

The early light microscopic lesions that appear in the bronchioles after 48 hours of oxygen inhalation are a ragged appearance of the mucosal layer, migration of the nucleus towards the lumen, and swelling of the cytoplasm (Harrison et al, 1970). Degeneration and necrosis of the mucosal 
cells then begin. The early changes that can be detected by transmission electron microscopy (Ludwin et al, 1974) are oedema of the epithelium, membranous bleb formation on the luminal surfaces, and loss of cilia after 72 hours of oxygen exposure. According to the studies on cultured human neonatal respiratory epithelium (Boat et al, 1973), cessation of ciliary movement and mucous clearance were related to squamous metaplasia or to degeneration and sloughing of epithelial cells, which occurred after 48-96 hours of exposure to $80 \%$ oxygen.

In our present investigation the lesions seen after 48 hours of exposure were denudation of cilia, a truncated shape of the remaining cilia, and flattening of the epithelial luminal surfaces. Bleb formation, moth-eaten appearance of the epithelial surfaces, loss of cilia in the bronchioles, and epithelial cell desquamation in the bronchi were also present after 96 hours of oxygen exposure. Reactions of the lung after exposure to high concentration of oxygen have been reported to vary with species and age (Kaplan et al, 1969; Norman et al, 1971; Goda, 1975), however, our results are in accord with other reported findings (Harrison et al, 1970; Ludwin et al, 1974) and it is suggested that bleb formation and moth-eaten appearance are the degenerative and necrotic processes of the epithelium.

Functioning cilia play an important part in the removal of various inhaled substances from the airway epithelial surface (Kilburn, 1968). Inhalation of high concentrations of oxygen suppresses mucociliary function (Sackner et al, 1976) even when pathological findings are not yet evident and administration of $100 \%$ oxygen is reported to produce a $45 \%$ decrease in tracheal mucous velocity in two hours. We would assume that the cilia cannot perform their sweeping action satisfactorily when ciliary damage has occurred.

Bronchiolar morphology differs between experimental animals (Hansell and Moretti, 1969; Castleman et al, 1975; Mariassy et al, 1975), and the protruding non-ciliated cells seen on bronchiolar surfaces are commonly termed Clara cells (Azzopardi and Thurlbeck, 1969; Cutz and Conen, 1971; Etherton et al, 1973; Kuhn et al, 1974; Petrik and Collett, 1974; Smith et al, 1974). Whether there is analogy between the mouse and human bronchiole or not is a problem. However, the protruded appearance of bronchiolar nonciliated cells, which are similar to the Clara cells of rodents, has been described in premature infants (Rosan and Lauweryns, 1972) and human adults (Ebert and Terracio, 1975). The role of Clara cells in the human and mammalian lung remains obscure, but several reports suggest that Clara cells could be the source of pulmonary surfactant (Niden, 1967) or, on the other hand, the sources of the basal layer of the alveolar lining (Cutz and Conen, 1971; Petrik and Collet, 1974). Recent studies, however, suggest that the secretion of Clara cells, possibly by an apocrine mechanism, provides a surface-active material to ensure their stability during pressure changes in the respiratory cycle (Maklem et al, 1969-70). It is thought that the atelectasis of the lung seen after exposure to high concentrations of oxygen is produced by bronchiolar collapse and mucous retention due to mucociliary dysfunction.

According to our earlier investigations (Goda, 1975), after mice have been returned to room air after exposure to high concentrations of oxygen, lung surface tension activity recovers to normal in three days, but microscopic lesions of the lung do not recover in 15 days because of lung fibrosis and capillary congestion. Our present study suggests that normal surface morphology does not recover in the bronchi and bronchioles in 15 days. But after the return to room air, the mice became cyanotic in our present experiments and the mortality rate increased considerably within three days. Therefore it is assumed that airway lesions of the early recovery phase were caused not only by the high concentration of oxygen, but also by hypoxia after the return to room air. From our present studies, we do not know how long it takes damaged airway epithelium to recover and further investigations will be needed to clarify this.

\section{References}

Adamson, I Y R, Bowden, D H, and Wyatt, J P O (1970). Oxygen poisoning in mice. Ultrastructural $₹$ and surfactant studies during exposure and recovery. 을

Archives of Pathology, 90, 463-472.
Anderson, W R, and Strickland, M B (1971). Pul- $\frac{D}{2}$ monary complications of oxygen therapy in the neonate. Postmortem study of bronchopulmonary $\mathcal{N}$ dysplasia with emphasis on fibroproliferative $N$ obliterative bronchitis and bronchiolitis. Archives of Pathology, 91, 506-514.

Azzopardi, A, and Thurlbeck, W M (1969). The O histochemistry of the non-ciliated bronchiolar 0 epithelial cell. American Review of Respiratory ${ }_{\mathbb{D}}^{\stackrel{D}{D}}$ Disease, 99, 516-525.

Banerjee, C K, Girling, D J, and Wigglesworth, J S (1972). Pulmonary fibroplasia in newborn babies treated with oxygen and artificial ventilation. $\vec{\Phi}$ Archives of Disease in Childhood, 47, 509-518.

Boat, T F, Kleinerman, J I, Fanaroff, A A, and $\stackrel{\mathbb{Q}}{2}$ Matthews, L W (1973). Toxic effects of oxygen on cultured human neonatal respiratory epithelium. Pediatric Research, 7, 607-615. 
Castleman, W L, Dungworth, D I, and Tyler, W S (1975). Intrapulmonary airway morphology in three species of monkeys. American Journal of Anatomy, 142, 107-122.

Cutz, E, and Conen, P E (1971). Ultrastructure and cytochemistry of Clara cells. American Journal of Pathology, 62, 127-134.

Ebert, R V, and Terracio, M J (1975). Observation of the secretion on the surface of the bronchioles with the scanning electron microscope. American Review of Respiratory Disease, 112, 491-496.

Etherton, J E, Conning, D M, and Corrin, B (1973). Autoradiographical and morphological evidence for apocrine secretion of dipalmitoyl lecithin in the terminal bronchiole of mouse lung. American Journal of Anatomy, 138, 11-36.

Goda, R (1975). Recovery processes from oxygen toxicity of lung in mice. Journal of the Japanese Association for Thoracic Surgery, 23, 1298-1309.

Hansell, M M, and Moretti, R L (1969). Ultrastructure of the mouse tracheal epithelium. Journal of Morphology, 128, 159-163.

Harrison, G, Rosan, R C, and Sloane, A (1970). Bronchiolitis induced by experimental acute and chronic oxygen intoxication in young adult rats. Journal of Pathology, 102, 115-122.

Kaplan, H P, Robinson, F R, Kapanci, Y, and Weibel, E R (1969). Pathogenesis and reversibility of the pulmonary lesions of oxygen toxicity in monkeys. I. Clinical and light microscopic studies. Laboratory Investigation, 20, 94-100.

Kilburn, K H (1968). A hypothesis for pulmonary clearance and its implications. American Review of Respiratory Disease, 98, 449-463.

Kistler, G S, Caldwell, $\mathbf{P} \mathbf{R} \mathbf{B}$, and Weibel, E R (1967). Development of fine structural damage to alveolar and capillary lining cells in oxygen-poisoned rat lung. Journal of Cell Biology, 32, 605-628.

Kuhn, C, Callaway, L A, and Askin, F B (1974). The formation of granules in the bronchiolar Clara cells of the rats. I. Electron microscopy. Journal of Ultrastructure Research, 49, 387-400.

Ludwin, S K, Northway, W $\mathbf{H}$, and Bensch, $\mathbf{K} \mathbf{G}$ (1974). Oxygen toxicity in the newborn. Necrotizing bronchiolitis in mice exposed to 100 per cent oxygen. Laboratory Investigation, 31, 425-435.

Maklem, P T, Proctor, D F, and Hogg, J C (196970). The stability of peripheral airways. Respiration Physiology, 8, 191-203.

Mariassy, A T, Plopper, C G, and Dungworth, D L (1975). Characteristics of bovine lung as observed by scanning electron microscopy. Anatomical Record, 183, 13-26.

Nash, G, Blennerhassett, J B, and Pontoppidan, $\mathbf{H}$ (1967). Pulmonary lesions associated with oxygen therapy and artificial ventilation. New England Journal of Medicine, 276, 368-374.

Niden, A (1967). Bronchiolar and large alveolar cell in pulmonary phospholipid metabolism. Science, 158, 1323-1324.

Norman, J N, Macintyre, J, Ross, R R, and Smith, G (1971). Etiological studies of pulmonary oxygen poisoning. American Journal of Physiology, 220, 492-498.

Northway, W H, Rosan, R C, and Porter, D Y (1967). Pulmonary disease following respiratory therapy of hyaline-membrane disease. Bronchopulmonary dysplasia. New England Journal of Medicine, 276, 357-368.

Northway, W H, Rosan, R C, Shahinian, L, Castellino, $R$ A, Gyepes, $M$ A, and Durbridge, T (1969). Radiologic and histologic investigation of pulmonary oxygen toxicity in newborn guinea pigs. Investigative Radiology, 4, 148-155.

Petrik, P, and Collet, A J (1974). Quantitative electron microscopic autoradiography of in vivo incorporation of $\mathrm{H}^{3}$-Choline, $\mathrm{H}^{3}$-Leucine, $\mathrm{H}^{3}$-Acetate and $\mathrm{H}^{3}$ Galactose in non-ciliated bronchiolar (Clara) cells of mice. American Journal of Anatomy, 139, 519534.

Rosan, R C, and Lauweryns, J M (1972). Mucosal cells of the small bronchioles of prematurely born human infants (600-1700 g). Beiträge zur Pathologie, 147, 145-174.

Sackner, M A, Hirsh, J A, Epstein, S, and Rywlin, A M (1976). Effect of oxygen in graded concentrations upon tracheal mucous velocity. Chest, 69, 164-167.

Smith, P, Heath, D, and Moosavi, H (1974). The Clara cell. Thorax, 29, 147-163.

Tsai, S H, Anderson, W R, Strickland, M B, and Pliego, M (1972). Bronchopulmonary dysplasia associated with oxygen therapy in infants with respiratory distress syndrome. Radiology, 105, 107112.

Requests for reprints to: Dr H Obara, Department of Anaesthesia, Kobe University School of Medicine, Kusunokicho-7, Ikutaku, Kobe, Japan 650. 